\title{
INCREASING INCIDENCE OF MULTIDRUG RESISTANCE Klebsiella pneumoniae INFECTIONS IN HOSPITAL AND COMMUNITY SETTINGS
}

\author{
SAVITA JADHAV*, RABINDRANATH MISRA, NAGESHAWARI GANDHAM, MAHADEV UJAGARE, \\ PURBASHA GHOSH, KALPANA ANGADI AND CHANDA VYAWAHARE
}

Department Of Microbiology, Pad. Dr. D. Y. Patil Medical College, Pimpri-411 018, Pune, MS, India

*Corresponding Author: Email- patilsv78@gmail.com

Received: June 22, 2012; Accepted: July 03, 2012

\begin{abstract}
-
Introduction- Klebsiella pneumoniae have been identified as an important common pathogen for nosocomial pneumonia (7 to $14 \%$ of all cases), septicaemia (4 to $15 \%$ of all cases), wound infections (2 to $4 \%$ of all cases), neonatal septicaemia ( 3 to $30 \%$ of all cases). It also causes bacteremia and hepatic infections and have been isolated from a number of unusual infections including endocarditis, primary gascontaining mediastinal abscess, cholecyctitis, diarrhoea, peritonitis, crepitant myonecrosis, pyomyositis, necrotizing fasciitis, osteomyelitis, meningitis. $\beta$-lactam antimicrobial agents are most common treatment option for such infections.

Results and observations- 986 Clinical isolates of Klebsiella pneumonia were isolated from blood (254), urine (241), respiratory specimens (206), pus (215) and body fluids (70). Among 986 clinical isolated of $K$. pneumoniae $718(72.81 \%)$ isolates were from inpatients (IPD) admitted in various wards of hospital and $268(27.18 \%)$ were found to be out patients department (OPD). A significant difference in antimicrobial

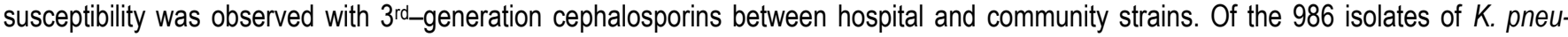
moniae $284(28.80 \%)$ were confirmed as ESBLs producers by phenotypic detection methods of ESBLs. Among 284 ESBLs 236 (83.09\%) were hospital isolates while $48(16.90 \%)$ were from community settings. Among 986 K. pneumoniae isolates 34 (3.44\%) strains showed production of KPCs by phenotypic detection methods of KPCs. Out of 34 KPCs producers 26 were from hospital isolates and 8 were from community isolates. Hospital isolates were major ESBLs and KPCs producers.

Conclusion- There is a serious need to accentuate on the rational use of antimicrobials and strictly adhere to the concept of the "reserve drug" to minimize the misuse of available antimicrobials. In addition regular antimicrobials susceptibility surveillance, knowledge and its application is essential to reduced current drug resistance rate in hospital as well as in community.
\end{abstract}

Key words- Klebsiella pneumonia, Extended spectrum $\beta$-lactamases, Klebsiella pneumonia carbapenemases, community isolates, hospital isolates.

Citation: Savita Jadhav, et al. (2012) Increasing Increasing Incidence of Multidrug Resistance Klebsiella pneumoniae Infections in Hospital and Community Settings. International Journal of Microbiology Research, ISSN: 0975-5276 \& E-ISSN: 0975-9174, Volume 4, Issue 6, pp.253-257.

Copyright: Copyright@2012 Savita Jadhav, et al. This is an open-access article distributed under the terms of the Creative Commons Attribution License, which permits unrestricted use, distribution and reproduction in any medium, provided the original author and source are credited.

\section{Introduction}

Klebsiella pneumoniae (K. pneumoniae) is clinically the most important member of Klebsiella genus of Enterobacteriaceae. Klebsiella pneumoniae have been identified as an important common pathogen for nosocomial pneumonia (7 to $14 \%$ of all cases), septicaemia (4 to $15 \%$ of all cases), wound infections (2 to $4 \%$ of all cases), neonatal septicaemia (3 to $30 \%$ of all cases) [1]. It also causes bacteremia and hepatic infections and have been isolated from a number of unusual infections including endocarditis, primary gas-containing mediastinal abscess, cholecyctitis, diarrhoea, peritonitis, crepitant myonecrosis, pyomyositis, necrotizing fasciitis, osteomyelitis, meningitis. They are also important opportunistic 
pathogens [2,3]. Clinical isolates of $K$. pneumoniae are generally resistant to a wider range of antibiotics, and virtually always naturally resistance to ampicillin and amoxycillin. $\beta$-lactam antimicrobial agents are most common treatment option for such infections. The persistent exposure of bacterial strains to a multitude of $\beta$ lactam has induced dynamic and continuous production and mutation of $\beta$-lactamases in these bacteria, expanding their activity even against newly developed $\beta$-lactam antibiotics and called as extended spectrum $\beta$-lactamases (ESBLs). K. pneumoniae and $E$. coli are remain the major ESBL-producing organisms isolated worldwide which are recommended to be routinely tested for and reported by CLSI (Clinical Laboratory Standards Institute) guidelines $[4,5]$.

ESBL prevalence rates among Indian hospitals vary greatly. First documentation of ESBL producing strains was made available on 15 strains of K. pneumonia in a medical centre at Vellore, South India [2]. The exact magnitude of the problem posed by ESBLs in Indian hospitals is not known in view of the fact that most of the laboratories routinely don't detect them. Carbapenem antibiotics, such as meropenem and imipenem have been the corner stone of drug treatment for serious infections caused by ESBls but developed a novel mechanism of resistance to carbapenem known as Klebsiella pneumoniae carbapenemases (KPCs) [6-9]. KPCs were first reported in 2001 in clinical isolate of Klebsiella pneumoniae from North Carolina [6-9]. Enzymes present in KPCs producing bacteria are also inactivate all penicillins, cephalosporins, azetreonam fluoroquinolones, aminoglycosides and most important carbapenems [9-12].

Several outbreaks have been documented by KPC including North Carolina, China, S. America, France, Israel. The plasmid that harbors the KPC resistance gene has transmitted to other Gram negative bacteria from Enterobacteriaceae like Klebsiella oxytoca, Escherichia coli, Serratia marcinens and non-fermenter group Pseudomonas spp [13-16]. Colistin and tigecycline are two drugs that have activity against KPCs with restrictions. Colistin causes neurotoxicity and nephrotoxicity while in vivo tigecycline use in such infections is not effectual due to its low drug blood levels [17-19]. Since ESBLs pose a major problem for clinical therapeutics, so it is obligatory to identify the prevalence of these strains in hospitals and characterize their epidemiology to control the spread of these strains to determine suitable preventive measures and treatment policies, the present study was undertaken in Pad. Dr. D. Y. Patil Medical College Pimpri-Pune-18.

\section{Materials and Methods}

The study was approved by the Institute Ethical committee and conducted between Jan 2006 to December 2011. The clinical specimen were obtained from inpatients and outpatients of the hospital, received from various specialties like medicine, surgery, obstetrics and gynecology, pediatrics, orthopedics, plastic surgery, dermatology, neurology, nephrology, cardiology and intensive care units. The repeat isolate from the same patient were excluded from the study. Clinico-demographic data of study patients was noted. The specimens were processed according to standard conventional bacteriological methods and bacterial pathogens were identified and confirmed by standard biochemical tests [20].
Antibiotic Susceptibility Tests- The Kirby- Bauer method recommended by the CLSI guidelines (2005) was used for antimicrobial susceptibility testing [20-22].

\section{Detection of Extended Spectrum $\beta$-Lactamases-Screening Test (CLSI, 2005)}

Initially screening test for ESBL production was done as part of routine susceptibility testing. Two antibiotic discs, ceftazidime (30 $\mu \mathrm{g})$ and cefotaxime $(30 \mu \mathrm{g})$ were used for screening for ESBLs. Plates with Mueller- Hinton Agar (MHA) were prepared and inoculated with the test organism (turbidity corresponding to $0.5 \mathrm{McFar}-$ land's standard) to form a lawn culture. The above discs were applied on the surface of the agar. The plates were incubated at $37^{\circ} \mathrm{C}$ overnight and sensitive pattern and resistant pattern were recorded by reading the zone diameter of the test organism. If a zone diameter of $\leq 22 \mathrm{~mm}$ for Ceftazidime and $\leq 27 \mathrm{~mm}$ for cefotaxime was recorded these strain were considered "Suspicious" for ESBL production [20-22].

\section{Double Disk Approximation Test (DDAT)}

Bacterial suspension equivalent to $0.5 \mathrm{McF}$ arland standards turbidity for testing ESBL production test were prepared. A sterile swab was dipped into standardized inoculum and the soaked swab was rotated against the upper inside wall of the tube to express excess fluid. The entire surface of the MHA was swabbed to form a lawn culture and the inoculum was allowed to dry for a minute with lid in place. With sterile forceps, Ceftazidime disk was placed on the agar plate near the centre giving a centre to centre distance of $15 \mathrm{~mm}$ Ceftazidime/clavulonic acid $(30 \mu \mathrm{g} / 10 \mu \mathrm{g})$. The plates were inverted and incubated at $37^{\circ} \mathrm{C}$ for $16-18$ hours. Each plate was examined for enhancement of zone of inhibition for ceftazidime disk at the side facing Ceftazidime/clavulonic acid disk. If the strain was an ESBL producer, then the zone around ceftazidime disk was extended towards Ceftazidime/clavulonic acid disk. ATCC Escherichia coli -25922 were used as negative control and ATCC K. pneumoniae -700603 was used as positive control [23-25].

\section{Detection of Metallo $\beta$-Lactamases by Imipenem-EDTA- Double Disk Synergy Test}

The IMP-EDTA combined disk test was performed as described by Yong et al._Test organisms were inoculated on to plates with MHA as recommended by the CLSI guidelines. Two $10 \mu \mathrm{g}$ imipenem disks (Hi Media, Mumbai India) were placed on the plate, and appropriate amounts of $10 \mu \mathrm{L}$ of $0.5 \mathrm{M}$ EDTA solution were added to one of them to obtain the desired concentration $(750 \mu \mathrm{g})$. The inhibition zones of the imipenem and imipenemEDTA disks were compared after 16 to 18 hours of incubation at $35^{\circ} \mathrm{C}$. In the combined disc test, if the increase in inhibition zone with the Imipenem and EDTA disc was $\geq 7 \mathrm{~mm}$ than the Imipenem disc alone, it was considered as MBL positive [24-26].

\section{Results and Observations}

986 Clinical isolates of Klebseilla pneumonia were isolated from blood (254), urine (241), respiratory specimens (206), pus (215) and body fluids (70). Year wise and specimen wise distribution are shown in Table 1.The repeat isolates were excluded from the study. Among 986 clinical isolated of K. pneumoniae 718 
$(72.81 \%)$ isolates were from inpatients (IPD) admitted in various wards of hospital and $268(27.18 \%)$ were found to be out patients department (OPD). A significant difference in antimicrobial susceptibility was observed with $3^{\text {rd }}$-generation cephalosporins between hospital and community strains. Antimicrobial susceptibility pattern of all clinical isolates was presented in Table 2. Amoxycillin showed decreased susceptibility in hospital isolates (17.40\%) and higher susceptibility in community isolates (64.17\%). Decreased susceptibility was also observed in tetracycline (17.54\%) and co-trimaxazole $(19.91 \%)$ in hospital isolates while cotrimaxazole showed high susceptibility in community isolates i.e. $(63.43 \%)$. In case of aminoglycosides, amikacin showed fair susceptibility both to hospital and community isolates i.e. $58.12 \%$ and $63.43 \%$ respectively. Gentamicin showed susceptibility $46.23 \%$ in hospital isolates and $56.71 \%$ in community isolates while amikacin showed better susceptibility than Gentamicin i.e. $58.21 \%$ and $63.43 \%$ in hospital and community isolates respectively.

Table 1. Year Wise and Specimen Wise Distribution of Klebseilla pneumoniae from Clinical Isolates

\begin{tabular}{|lllllll|} 
Year & Blood & Urine & Pus & Sputum & Others & Total \\
\hline 2007 & 38 & 30 & 20 & 21 & 9 & 118 \\
2008 & 44 & 32 & 24 & 28 & 20 & 148 \\
2009 & 58 & 45 & 38 & 35 & 11 & 187 \\
2010 & 69 & 62 & 63 & 56 & 10 & 260 \\
2011 & 45 & 72 & 70 & 66 & 20 & 273 \\
Total & 254 & 241 & 215 & 206 & 70 & 986 \\
\hline
\end{tabular}

Table 2- Antimicrobial Susceptibility of Clinical Isolates of Klebsiella pneumoniae

\begin{tabular}{|c|c|c|c|c|}
\hline \multirow[t]{2}{*}{$\begin{array}{l}\text { Name of } \\
\text { antibiotics }\end{array}$} & \multicolumn{2}{|c|}{$\begin{array}{l}\text { Hospital isolates [n=718 } \\
(72.81 \%)]\end{array}$} & \multicolumn{2}{|c|}{$\begin{array}{l}\text { Community isolates }[\mathrm{n}=268 \\
(27.18 \%)]\end{array}$} \\
\hline & Susceptibility (\%) & Resistance (\%) & Susceptibility & Resistance (\%) \\
\hline Amikacin & $418(58.21)$ & $300(41.78)$ & $170(63.43)$ & $98(36.56)$ \\
\hline Amoxycillin & $125(17.40)$ & 593 (82.59) & $172(64.17)$ & $96(35.82)$ \\
\hline Cefotaxime & 305 (42.47) & $413(57.97)$ & $220(82.08)$ & $48(17.91)$ \\
\hline Ceftriaxone & $421(58.63)$ & $297(41.36)$ & $202(75.37)$ & 66 (24.62) \\
\hline Ciprofloxacin & $341(47.49)$ & $377(52.50)$ & $212(79.10)$ & $56(20.89)$ \\
\hline Cotrimaxazol & $143(19.91)$ & $575(80.08)$ & $170(63.43)$ & $98(36.56)$ \\
\hline Ceftazidime & $460(64.06)$ & $258(35.93)$ & $209(77.98)$ & $59(22.01)$ \\
\hline $\begin{array}{l}{ }^{*} \text { Ceftazidime } \\
+ \text { Calvulanic } \\
\text { acid }(n=258)\end{array}$ & $236(91.47)$ & 22( & 48 & $11(18.64)$ \\
\hline Gentamycin & $332(46.23)$ & $386(53.76)$ & $152(56.71)$ & $116(43.28)$ \\
\hline Imipenem & 618 ( 86.07) & $100(13.92)$ & $233(86.94)$ & $35(13.05)$ \\
\hline $\begin{array}{l}\text { *Imipenem + } \\
\text { EDTA }(n=100)\end{array}$ & $26(26)$ & $74(74)$ & $8(22.85)$ & $27(77.14)$ \\
\hline Tetracycline & $126(17.54)$ & $592(82.45)$ & $98(36.56)$ & $170(63.43)$ \\
\hline
\end{tabular}

${ }^{*}$ Ceftazidime + Calvulanic acid disc susceptibility testing were done on only ceftazidime resistance strains. Imipenem + EDTA disc susceptibility testing were done on only Imipenem resistance strains.

All isolates were screened for detection of ESBLs and KPCs and shown in Table 3 and Table 4. Of the 986 isolates of K. pneumoniae $284(28.80 \%)$ were confirmed as ESBLs producers by phenotypic detection methods of ESBLs. Among 284 ESBLs 236 $(83.09 \%)$ were hospital isolates while $48(16.90 \%)$ were from community settings. Among 986 K. pneumoniae isolates 34 (3.44\%) strains showed production of KPCs by phenotypic detection meth- ods of KPCs. Out of 34 KPCs producers 26 were from hospital isolates and 8 were from community isolates. Hospital isolates were major ESBLs and KPCs producers. Year wise and sample wise distribution was depicted in Fig. 1. All $34 \mathrm{KPCs}$ producing isolates were susceptible to tigecycline and polymixin $\mathrm{B}$.

Table 3- Detection of ESBL Producers by Screening Test and Confirmatory Test.

\begin{tabular}{|llll|} 
Specimen & $\begin{array}{l}\text { ESBL producers by } \\
\text { screening test }\end{array}$ & $\begin{array}{l}\text { ESBL producers by DDAT } \\
\text { (confirmatory test) }\end{array}$ & $\begin{array}{l}\text { Non-ESBL } \\
\text { producers }\end{array}$ \\
\hline Blood & 83 & 74 & 180 \\
Urine & 77 & 64 & 177 \\
Pus & 61 & 53 & 162 \\
Sputum & 58 & 61 & 145 \\
Others & 38 & 32 & 38 \\
Total & 317 & 284 & 702 \\
\hline
\end{tabular}

Table 4- Ward Wise Distribution of Patients With ESBL and KPCS Producers Positive Isolates

\begin{tabular}{|llll|} 
Ward & $\begin{array}{l}\text { Positive isolates } \\
(\mathrm{n}=986)\end{array}$ & $\begin{array}{l}\text { ESBL producers } \\
(\mathrm{n}=284)\end{array}$ & $\begin{array}{l}\text { KPCs } \\
\text { producers }\end{array}$ \\
\hline Surgery & 153 & 62 & 6 \\
Medicine & 135 & 47 & 6 \\
I.C.U. & 51 & 30 & 1 \\
N.I.C.U. & 33 & 12 & 3 \\
P.I.C.U. & 17 & 8 & 1 \\
Pediatrics & 40 & 17 & 1 \\
Pulmonary medicine & 106 & 25 & 2 \\
OBGY & 75 & 13 & 2 \\
Urology & 55 & 12 & 2 \\
Orthopedics & 53 & 10 & 2 \\
Out patients & 268 & 48 & 8 \\
TOTAL & 986 & 284 & 34 \\
\hline
\end{tabular}

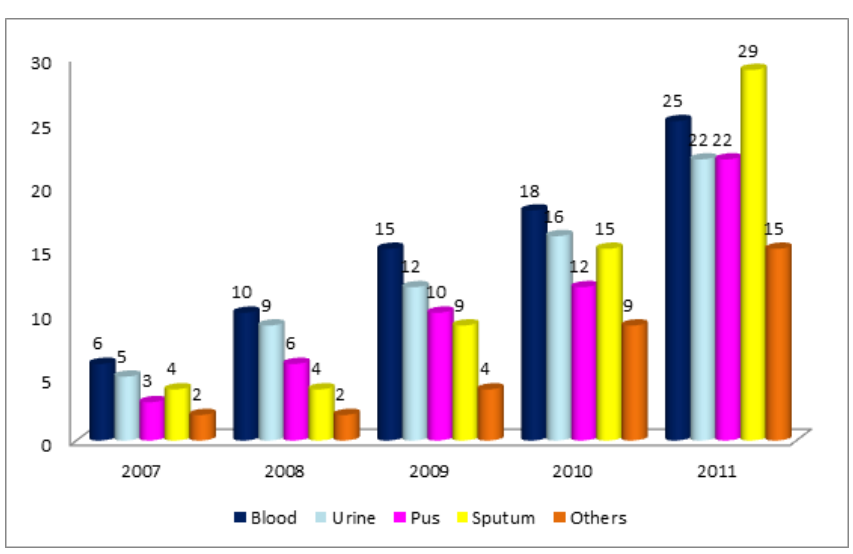

Fig. 1- Year Wise and Sample Wise Distribution of ESBL Producing Clinical Isolates of K. pneumonia.

\section{Discussion}

In recent years a significant increase in ESBL producing $K$. pneumoniae in hospitalized patients are problematic throughout world. Epidemiological data showed great variation in prevalence in European and Asian countries [27-29]. The emergence of the Klebsiella pneumoniae carbapenemases in $K$. pneumoniae and other Gram-negative bacteria, usually on a background of multidrug resistance, has led to difficult therapeutic choices. Among available antibiotics, tigecycline and the polymyxin $B$ are the most 
frequently active against these organisms in vitro. Optimal therapy of infections due to these bacteria may involve maximization of antibiotic dose as well as their use in combination [30-32].

$\mathrm{KPC}$-producing $\mathrm{K}$. pneumonia are also being reported more often. The first case of KPC-producing $K$. pneumoniae infection was reported in 2005 in France and had a US origin [33-35]. The first outbreak of KPC-producing $K$. pneumoniae outside the United States was in Israel [12]. In South America, dissemination of KPCproducing K. pneumoniae was initially reported in 2006 in Colombia [12] and then in Brazil and Argentina [12, 34]. KPC enzymes have also been identified in $P$. aeruginosa isolates from Colombia $[14,16]$. In the People's Republic of China, KPC enzymes in several enterobacterial species are being increasingly reported [16]. Finally, in Europe a few cases of KPC-producing K. pneumoniae infection have been described, but in Greece, outbreaks have occurred $[12,14,34]$. In Europe, different variants of KPCs (KPC-2 and KPC-3) have been described [16].

The current study demonstrates a prevalence of ESBLs and KPCs production by $K$. pneumoniae i.e. $28.80 \%$ and $3.44 \%$ respectively. $K$. pneumoniae with ESBLs production were more prevalent in Latin America (45\%), followed by the Western pacific region (24\%), Europe (22\%), the United State $(7.6 \%)$ and Canada $(4.9 \%)$ [6, 12]. In Middle East, Saudi Arabia (12.2\%), Lebanon (20\%), Egypt (37.5\%), Israel (79\%), and Turkey (50\%), Jordan (80\%) [3] and Pakistan $(36 \%)$ [10]. The acquisition of the multi drug resistant $K$. pneumoniae infection in our data showed that lower extreme of age, who have underdeveloped immunity and are therefore more likely susceptible to infections with opportunistic pathogens such as K. pneumoniae. In all ICUs, prevalence of ESBL and KPCs producers were also elevated because of their debilitated conditions, likely to need ventilatory assistance, and had greater exposure to various antimicrobial agents. Other risk factors such as male gender, intravascular and urinary catheterisation, mechanical ventilation, prior surgery. Most of these patients have been previously hospitalized at other institutions and subjected to different therapies including prenatal antibiotics. Because of the complicated nature of their conditions, many patients admitted have prolonged hospitalizations, several invasive procedures, and extensive antibiotic treatments.

All these factors may contribute to the patient's acquisition of multidrug resistant bacteria.

KPCs and ESBLs producers were found to be parallel resistant to broad spectrum antimicrobials classes, indicating that co-transfer of a genes accounting for resistance to these antibiotics. Various studies have reported increased isolation and increased rates of nosocomial infections with ESBL producing $\mathrm{K}$. pneumoniae in their neonatal and pediatrics patients both from developed and developing countries $[2,12,18,35]$.

Our study presents bacteraemic isolates from medicine and surgery wards were more likely to be associated with ESBLs and KPCs production as compared to non bacteraemic isolates [14]. Urinary isolates also have greater percentage in association of ESBLs and KPCs production. Nitrofurantoin showed excellent susceptibility to urinary isolated. All KPCs producing isolates from our data showed susceptibility to tigecycline and polymyxin B in vitro antimicrobial susceptibility testing. Most published experience of treatment of infection due to KPC-producing organisms is with tigecycline, a polymyxin (polymyxin B or colistin), or their combination, with variable reported outcomes. Monotherapy, however, may be associated with persistent infection and the emergence of antibiotic resistance. Recurrence of an empyema due to a KPC-producing K. pneumoniae during treatment with tigecycline was associated with an increase in MIC from 0.75 to $2.0 \mu \mathrm{g} / \mathrm{mL}$ [34]. Elemam and colleagues [8] recently reported two patients with infections due to $K$. pneumoniae isolates resistant to all antibiotics tested, including tigecycline and polymyxin $B$, with both isolates having developed progressive increases in the MIC to polymyxin $B$ during treatment with this antibiotic $[27,30]$.

Our data have certain limitations, as collected data were not from programmed survey of drug resistance but obtained from routine analysis of specimens submitted to the laboratory therefore is subjected to sampling bias. Secondly phenotypically confirmed ESBLs and KPCs isolates should have confirmed by molecular methods with specific primers to do characterization for understanding their molecular epidemiology and clinical outcome. Nevertheless, despite these limitations these observations provide some insight into the epidemiology and the clinical importance of this new threat: namely ESBLs and KPC-producing K. pneumoniae.

\section{Conclusion}

To manage the burden of ESBL-producing pathogens, suitable infection control interventions should be implemented for all patients who are infected or colonized with ESBL and KPCs producing bacteria. These interventions include effective hand hygiene and instituting contact precautions for all colonized and infected patients. However there is a serious need to accentuate on the rational use of antimicrobials and strictly adhere to the concept of the "reserve drugs" to minimize the misuse of available antimicrobials. In addition regular antimicrobials susceptibility surveillance, knowledge and its application is essential to reduced current drug resistance rate in hospital as well as in community.

\section{References}

[1] Podschun R. and Ullmann U. (1998) Clin. Microbiol. Rev., 11, 589-603.

[2] Purva M., Arti K., Bimal D., Benu D. (2002) Indian J. Med. Res., 115, 153-57.

[3] Rabindranath R.N. (2012) Medical Journal of Dr. D. Y. Patil University, 5 (1), 10-13.

[4] Jadhav Savita, Hussain A., Devi S., Kumar A., Parveen S. Gandham N., Wieler L.H., Ewers C. and Ahmed N. (2011) PLoS One 6, e18063.

[5] Avasthi T.S., Kumar N., Baddam R., Hussain A., Nandanwar N., Jadhav S. and Ahmed N. (2011) India. J. Bacteriol., 193, 4272-3.

[6] Livermore D.M. (1995) Clin. Microbiol. Rev., 8, 557-84.

[7] Desimoni M.C, Esquivel G.P, Merino L.A. (2004) Enferm. Infecc. Microbiol. Clin., 22, 507-511.

[8] Elemam A., Rahimian J. and Mandell W. (2009) Clin. Infect. Dis., 15,49(2), 271-274.

[9] Bush K., Jacoby G.A. and Medeiros A.A. (1995) Antimicrob. Agents. Chemother., 39, 1211-33.

[10]Jain A., Roy I., Gupta M.K., Kumar M., Agarwal S.K. (2003) J. Med. Microbiol., 52, 421-5.

[11]Shukla I. (2004) Indian J. Med. Microbiol., 22, 87-91. 
[12]Patterson D.L, Ko W.C., Gotberg A.V., Mohapatra S., Classels J.M., Goosness H. et al. (2004) Ann. Intern. Med.,140, 26-32.

[13]Gupta E., Mohanty S., Sood S., Dhawan B., Das B., Kapil A. (2006) Indian J. Med. Res., 124, 95-8.

[14]Kurokawa H., Yagi T., Shibta N. and Arakawa Y. (1999) Lancet, 354-955.

[15]Gaynes R.P., Culver D.H. (1992) Infect. Control. Hosp. Epidemiol., 13(1), 10-4.

[16]Nordmann P., Cuzon G. and Nass T. (2009) Lancet. Infect. Dis., 9(4), 228-36.

[17]Arnold R.S, Thom K.A, Sharma S., et al. (2011) South Med. J., 104(1), 40-5.

[18]Hansonita J.B., Agarwal V., Pathak A.A., Saoji A.M. (1997) Indian J. Med. Res., 105, 160-5.

[19]Manchanda V., Singh N.P., Goyal R., Kumar A., Thukral S.S. (2005) Indian J. Med. Res., 122, 330-7.

[20]Koneman E.W., Allen S.D., Janda W.M., Schreckenberger P.C., Win W.C. (1997) Color atlas \& textbook of diagnostic microbiology, $5^{\text {th }}$ ed. Philadelphia: JB Lippincott co. 171-230.

[21]Clinical and laboratory Standards Institute, Performance Standards for antimicrobial susceptibility testing.

[22]Anderson K.F., Lonsway and Rasheed J.K. (2007) Evaluation of methods to identify the Klebsiella pneumonia carbapenemase in Enterobacteriaceae, 45 (8), 2723-2725.

[23]Pitout J.D., Hossain, Hanson N.D. (2004) Journal of Clinical Microbiology, 42 (12), 2153-2162.

[24]Thomason K.S. and Sanders C.C. (1992) Antimicrobial agents and chemother, 36, 1877-1882.

[25]Lee k., Chong Y., Shin H.B., Kim Y.A., Yong D. (2001) Clin. Microbiol. Infect., 7, 88-89.

[26]Hirsch E.B. and Tam V.H. (2010) J. Antimicrob. Chemother., 65(6), 1119-25.

[27]Pournaras S., Vironi G., Neou E., et al. (2011) Int. J. Antimicrob. Agents, 37(3), 244-7.

[28]Pankey G.A and Aschcraft D.S. (2011) Diag. Microbiol. Infect. Dis., 70(4), 561-4.

[29]Bratu S., Tolaney P., Karumudi U., et al. (2005) J. Antimicrob. Chemother., 56(1), 128-32.

[30]Neuner E.A, Yeh J.Y, Hall G.S., et al. (2011) Diag. Microbiol. Infect. Dis., 69(4), 357-62.

[31]Gasink L.B., Edelstein P.H., et al. (2009) Infect Control Hosp. Epidemiol., 30(12), 1180-5.

[32]Patel G., Huprikar S., Factor S.H., et al. (2008) Infect Control Hosp. Epidemiol., 29(12), 1099-106.

[33]Lee J., Patel G., Huprikar S., et al. (2009) J. Clin. Micobiol., 47 (5), 1611-2.

[34]Steinmann J., Kaase M., Popp W., et al. (2011) Euro. Surveill., 16(33), 19944.

[35]Centre's for Disease Control and prevention (CDC) (2009)

MMWR Morb. Mortal Wkly. Rep., 20, 58(10), 256-60. 